\title{
Research Roadmap for Green Wireline Access
}

\author{
Peter Vetter \\ Bell Labs, \\ Alcatel-Lucent \\ Laurent Lefevre \\ INRIA, \\ Lyon, France
}

Murray Hill, NJ

\author{
Laurent Gasca \\ Draka \\ (Prysmian Group), \\ Marcoussis, \\ France
}

\author{
Konstantinos \\ Kanonakis \\ AIT, \\ Athens, Greece
}

\author{
Leonid \\ Kazovsky \\ Stanford \\ University, \\ Stanford, CA
}

Bart Lannoo
Ghent
University -
IBBT,
Ghent,
Belgium

Abstract-The paper describes different research concepts of which the combination leads to an improvement of the power consumption per subscriber in a wireline access network by 10x and the energy efficiency per transferred bit by 100x.

Keywords: wireline access; green networks; energy efficiency; PON; virtual home gateway

\section{INTRODUCTION}

The purpose of this paper is to provide a research roadmap of energy saving concepts for wireline access networks and assess their respective effect on the total average power consumption per subscriber. The roadmap was elaborated by the Wireline Access working group of GreenTouch [1]. GreenTouch is a global research consortium aiming at several orders of magnitude reduction of the energy efficiency in different parts of the network. The wireline access network is the second largest consumer of energy after wireless networks and hence an important focus area for efficiency improvements [2].

About $80-90 \%$ of the energy in today's wireline networks is consumed in the access network, with about $10 \mathrm{~W}$ per user being dissipated mostly by the customer premises equipment (CPE). The estimated energy needed to provide network access to the 100 million U.S. households amounts to $10 \mathrm{TWh}$, which is equivalent to the emission of 6 million tons of $\mathrm{CO} 2$ per year (assuming $0.67 \mathrm{~kg} / \mathrm{kWh}$ ). In addition to the carbon footprint issue, power consumption in access systems has historically represented a problem for network operators due to the cost of power supply in remote units, heat dissipation in high density access nodes of the central offices and requirements for backup battery capacity for lifeline service support during periods of power outage. The current engineering approaches to minimizing the power consumption, such as ASIC integration, migration to smaller scale CMOS technologies, efficient cooling, dynamic power management and sleep modes, will not be sufficient to keep the power consumption and $\mathrm{CO}_{2}$ emission at the current level considering the exponential growth of traffic in the future [2]. The need for dramatic improvement in

Xing-Zhi
Qiu
IMEC,
Ghent,
Belgium

Fabienne

Saliou

Orange Lab,

Lannion,

France
Adriaan

Wonfor

University of

Cambridge,

Essex, UK

energy efficiency will mandate disruptive changes in the implementation of access networks.

The access network offers some unique opportunities for network efficiency improvements:

- Shorter range and lower rate, which offer a different sweet-spot for innovation of energy efficient transmission than in optical core networks;

- Tree topology with mux-demux aggregation, as opposed to many-inputs-to-many-outputs forwarding like in core routers;

- High underutilization of electronic processing due the fact that fewer users share the same equipment than in a core network, which offers opportunities to exploit dynamic power management and sleep modes;

- Lower processing rates than the core network;

- Application awareness at a subscriber level may allow for application specific energy saving concepts.

By the year 2020, the research performed by the Wireline Access working group of the GreenTouch Consortium is expected to lead to the deployment of access networks featuring a more than 10 times lower power consumption per user and 10 times higher bit rates, compared to the current state-of-the-art wireline access networks. The resulting energy efficiency per bit is therefore expected to be improved by a factor of 100 .

The paper describes the research challenges and targets to achieve this overall objective. Section II starts with the baseline and the methodology used to calculate the power consumption per subscriber and the energy efficiency. Section III contains the actual roadmap with a summary of short, medium, and long term concepts. Section IV concludes the paper and presents an outlook of the ongoing research in the Wireline Access working group of GreenTouch. 


\section{BASELINE ARCHITECTURE AND MODELLING METHODOLOGY}

\section{A. Baseline architecture}

Our baseline architecture is a fiber-to-the-home (FTTH) network using gigabit capable passive optical network (GPON) [3], as well as the recently standardized ten gigabit capable passive optical network (XG-PON1) [4]. It is acknowledged that the power consumption for the IEEE variants, EPON and 10 GEPON is very similar and hence the same conclusions for the roadmap are valid. Other FTTX architectures with digital subscriber line (DSL) or hybrid fiber coax (HFC) are widely deployed, but we decided to concentrate on FTTH, because it is the most energy-efficient wireline access technology today. Within the options for FTTH, we focus on PON, because it is widely deployed and consumes the least power when considering today's standards. In a parallel effort, we are addressing the energy efficiency of other access architectural options, as introduced in [5].

\section{B. Model for power consumption}

We estimated the power consumption for the optical line termination (OLT) in the central office (CO), and the optical network unit (ONU) (also called customer premises equipment (CPE)). The OLT includes the first mile line termination, packet processing and traffic management, layer 2 (L2) aggregation, and interface to the metro aggregation network. In order to calculate the power per user of the OLT, we assumed $50 \%$ filling of a PON with a splitting factor of 64 , in other words 32 homes connected. The CPE includes the termination of the first mile at the subscriber end, wired and wireless interfaces to the local area network (LAN), and processing for layer 3 (L3) routing and home gateway functionality. Since a major part of the power consumption for wireline access networks is at the CPE, we further divided the CPE in its subsystems in order to assess the effect of different new energy saving concepts on the respective parts (cf. Table 1).

TABLE I. BREAKDOWN OF SUBSYSTEMS IN CPE

\begin{tabular}{|l|l|}
\hline Subsystem & Functional blocks included \\
\hline EO PON & $\begin{array}{l}\text { Downstream Receiver } \\
\text { Upstream Transmitter }\end{array}$ \\
\hline PON Digital & $\begin{array}{l}\text { Digital electronics to terminate PON, medium access } \\
\text { control (MAC), forward error coding (FEC), and layer } \\
2 \text { switching }\end{array}$ \\
\hline Wireline LAN & 4x Gigabit Ethernet \\
\hline $\begin{array}{l}\text { pome gateway } \\
\text { processor }\end{array}$ & $\begin{array}{l}\text { Processor for operation, administration and } \\
\text { maintenance (OAM), routing, network address } \\
\text { translation (NAT), and security functions }\end{array}$ \\
\hline (Wireless LAN) & Wi-Fi 802.11n as baseline reference \\
\hline
\end{tabular}

We started from the target figures for wireline access defined for 2011-2012 by the European Code of Conduct (EU

\footnotetext{
${ }^{1}$ Note: we include this for completeness, but have no specific research activities in the Wireline Access working group to improve the efficiency of the WLAN interface. This is considered part of the Mobile working group in GreenTouch.
}

CoC) [6], to which several GreenTouch member organisations have contributed. When the figures in the $\mathrm{EU} \mathrm{CoC}$ did not provide sufficient detail, we estimated the power consumption for functional blocks using recent datasheets published by component vendors on the Internet in the time frame of 20092011. Datasheet values are assuming a specified DC voltage supply. We factored in an efficiency of $80 \%$ for the power supply. For new concepts, we estimated the improvement in percentages of the current value. More accurate estimations will be available as GreenTouch progresses. In some cases, we estimated the power by designing the specific new functions and simulating them with hardware design tools.

\section{Energy efficiency}

In order to evaluate the contribution of the Wireline Access working group towards the GreenTouch objectives, we also calculated the energy efficiency per bit in target reference year 2020. We compared it to a business as usual (BAU) trend, which considers user rate evolution and energy efficiency gains without specific measures addressed in GreenTouch, such as Moore's law and hardware design improvements.

Specifically for the access network, we defined the energy efficiency per bit as the average power consumption per home divided by the sustained subscriber rate during busy hour. We started from an average subscriber rate of $10 \mathrm{Mbit} / \mathrm{s}$ in 2010 and assumed that this rate will double every 5 years, in other words increase by a factor of $4 \mathrm{x}$ by 2020 . This results in an average subscriber rate of $40 \mathrm{Mbit} / \mathrm{s}$ by the end of the decade, or the equivalent of 4 HDTV streams for a single home. The average may include long lasting sessions of streaming services and higher instantaneous peak rates of 1 or $10 \mathrm{Gbit} / \mathrm{s}$ for fast downloads during short periods of time (minutes or seconds). The calculation of the energy efficiency is highly dependent on the assumptions made for the evolution of the subscriber rate. We therefore included a sensitivity analysis by observing the efficiency gain for different subscriber rate evolutions.

\section{ROADMAP}

Figure 1 shows the power consumption per subscriber of a wireline access network and the expected improvement per energy saving concept.

As a roadmap, we classified the research concepts as:

- Short term: commercialisation in first products can already be expected by the end of GreenTouch in 2015.

- Medium term: commercialisation can be expected a few years after the end of GreenTouch. After a proof of concept within GreenTouch, one should typically count two to three years of development before first products are available. The concepts are not dependent on other boundary conditions for their commercialisation, e.g. a standard is already in place or there is no need for standardisation.

- Long term: these concepts are so disruptive that they require additional actions and changes in the market place: new standarisation (e.g. a new protocol or network architecture), roll-out of a new infrastructure 
(e.g. single mode fiber in the home), or adoption of new technologies by the industry (e.g. low power electronics). Their commercialisation may take about a decade or longer from now.

The reference is GPON in 2010, which is the year the research activities in GreenTouch started. First XG-PON1 products are released in 2012, which is about two years after the approval of the standard. Many of the innovations focus on reducing the power consumption of the electronic circuitry, such as sleep-mode or new protocols, because the electrooptical front end is only $10 \%$ of the total power. The green labels in Figure 1 show the research concepts addressed by GreenTouch. The white labels indicate improvements that are happening outside of GreenTouch.

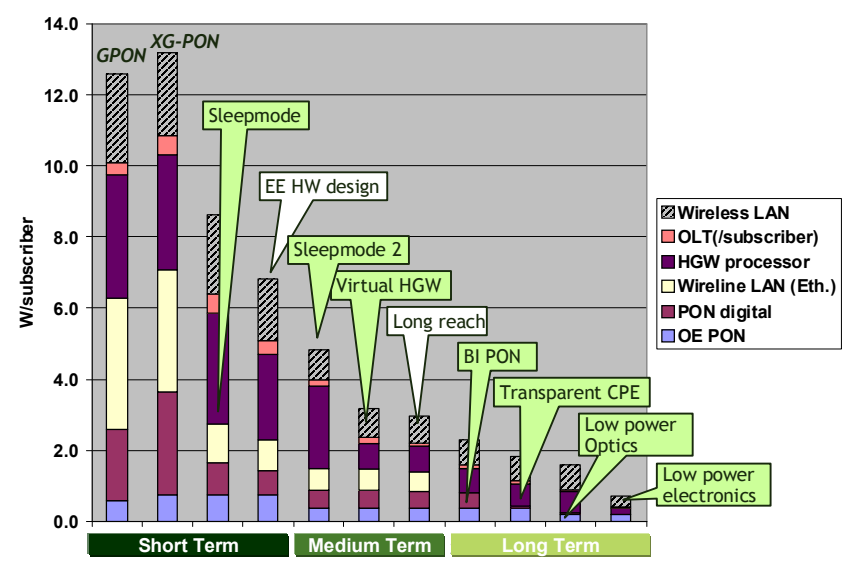

Figure 1. Power consumption improvements in a wireline access network.

Figure 1 clearly illustrates how the different concepts affect the power consumption of a specific subsystem. The contribution of the Wireless LAN interface of the CPE is added for completeness, but is not within the scope of the Wireline Access working group. Figure 1 shows that the combination of research concepts will reduce the power per subscriber more than 10x compared to the baseline architecture.

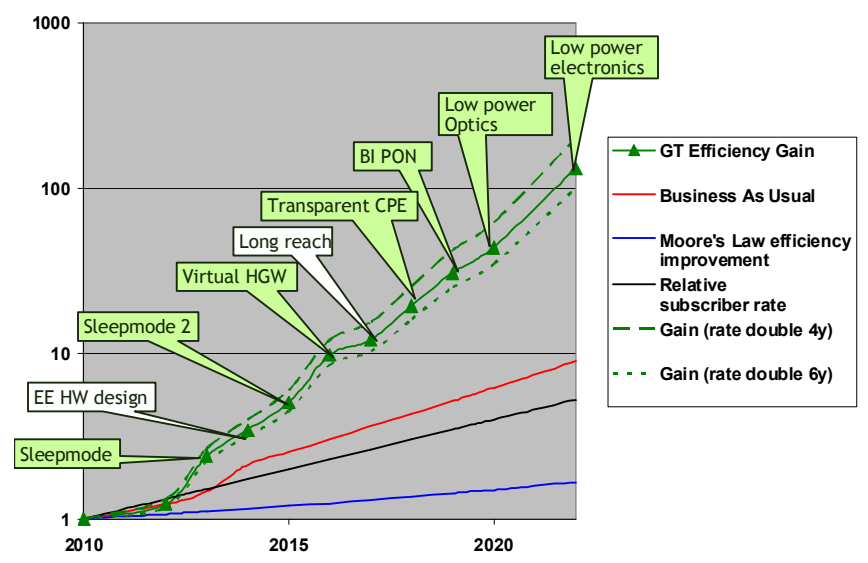

Figure 2. Evolution of energy efficiency per bit for wireline access.
Figure 2 shows the evolution of the energy efficiency per bit resulting in a gain of about $100 \mathrm{x}$ around 2020. The introduction year in which a concept is expected to be commercially available is speculative and for illustration purposes only. Changing the year or order does not influence the calculation of the efficiency gain assuming availability in 2020. The BAU trend includes Moore's law and concepts outside of GreenTouch, such as HW energy efficiency improvements and long reach PON. We assumed that the subscriber rate doubles every 5 years, but also performed a sensitivity analysis for doubling every 4 years and every 6 years.

\section{A. Short term}

Sleepmode is an important concept to reduce the average power consumption of a CPE, because the CPE is not always used at the maximum throughput capacity that it is designed for and hence idling for a large part of the time. A coarse type of sleepmode is hibernation at times of the day or night that the subscriber is not connected. A next level of sleepmode is power shedding of functional blocks (e.g. specific interfaces) that are not in use and only turned on when a session is established. These types of sleep modes are already technically feasible today and help to lower the energy consumption averaged over longer periods of time (they may however not yet be implemented in currently deployed CPE). Recent standards [4] have defined sleep cycles on XG-PON1 over a time scale of tens of milliseconds and allow for power savings while communication sessions are active. Energy Efficient Ethernet achieves similar savings on the LAN interface [7]. The power consumption of digital electronics in CPE will benefit from these concepts on the short term.

Energy Efficient Hardware design (EE HW) refers to engineering optimisations and can still be considered a short term evolution. It is estimated that they bring about $20 \%$ efficiency improvements to the original design. A first optimisation is to reduce the transfers of data across $\mathrm{I} / \mathrm{O}$ of ASICs thanks to further integration, new interconnect techniques like multi-chip modules, and a critical review of the number of required memory accesses. A second optimisation is the use of clock gating when a functional block in a digital circuit is not active. Other methods to be considered are dynamic voltage and frequency scaling (DVFS) and asynchronous designs.

\section{B. Medium Term}

Sleepmode 2 groups more advanced sleepmode concepts, such as designs that allow for a faster turn-on time or a lower power consumption during a sleep state, which will allow for additional savings on the medium term. While the earlier sleepmode capabilities are mainly focused on CPE, we can also expect dynamic load management concepts for the access node (OLT) on the medium term.

Virtual Home Gateway (VHG) runs the typical home gateway functions, such as routing, OAM, and security, on a central server in the network instead of a processor on the CPE (cf. Figure 3). Unlike the short sleep periods applied to data transfer functions in a CPE, it is more difficult to apply aggressive sleep cycles with periodicity of milliseconds on a 
central processing unit (CPU) and hence, the $\mathrm{CPU}$ remains powered on in a conventional $\mathrm{CPE}$. Thanks to the virtualisation, the $\mathrm{CPU}$ in the $\mathrm{CPE}$ can be reduced or even eliminated, while the power at the server is more efficiently used thanks to the time sharing of a single processor on a server by multiple subscribers. Routing, which is currently performed in the processor on a CPU, can be offloaded to more efficient router hardware in the network. A preliminary estimate is a factor of three saving of the power for the processing (further research is required to obtain a more accurate number). Other motivations for an operator to deploy VHG are easier operation and maintenance on a central server than on distributed devices. It also facilitates the upgrade of new services that require more processing capability than available on $\mathrm{CPE}$ deployed in the field. It extends the lifetime and management of wireline CPE by reducing the complexity of deployed equipments. VHG can already be used for simple connectivity boxes (e.g. Ethernet L2 CPE) on the medium term, because it does not require additional standardisation. It can be combined with a transparent CPE concept in the longer term, as described below.

Long reach access architectures reduce the number of hops in the access and aggregation network. A SuperPON with split of about 1:512-2048 and a range of $100 \mathrm{~km}$ allows for bypassing the local exchange. Although the OLT function is not eliminated, but moved up in the network to the edge location, some of the electronic processing for aggregation and regeneration of data could be removed. Despite an additional power to achieve optical budget extension, the estimated net saving is about $50 \%$ on the networks side. Reach extender boxes are already defined in the standards, but lower cost solutions and cost effective redundancy schemes are required to make this solution commercially viable on the medium term.

\section{Long Term}

Bit Interleaving PON is a new time division multiplexing (TDM) transfer protocol under development in GreenTouch that reduces the power consumption of the PON digital part for the downstream by almost an order of magnitude. In a conventional packet based PON system, every ONU processes all downstream data until it is able to extract the incoming packets destined for the local area network and drop the rest of the data. A lot of power is consumed in the processing of this high throughput (e.g. $10 \mathrm{Gbit} / \mathrm{s}$ in XG-PON1). The new concept of bit interleaving allows for selecting the relevant bits immediately behind the clock and data recovery. Further processing is done at the user rate instead of the aggregate line rate, which results in significant power savings. Bit Interleaving does not work for upstream. We propose to use a similar upstream burst mode transmission as for a conventional PON is used, because it is already energy efficient due to its load proportionality. This is a long term concept, because standards for XG-PON1 and 10GEPON have recently been endorsed and the industry is expected to capitalize on these efforts before being ready for a new specification.

Transparent CPE is a disruptive concept that provides a completely passive connectivity between the first mile and the home network (cf. Figure 3) [8]. The functions of conventional $\mathrm{CPE}$ can be moved to a server in the network by the virtual home gateway (VHG) concept described above. With the emergence of easy connectorisation and flexible bend single mode fiber for in-house wiring, it will be conceivable to have an optically transparent link across the home and access networks. The actual implementation with e.g. WDM or passive splitting is still subject of research. It is also acknowledged that there will not be a single mode fiber to every device in the home. We anticipate a cut-through connectivity via single mode fiber to high bandwidth devices (e.g. TV or multimedia terminals), while maintaining a wireless LAN for low bandwidth devices (e.g. sensors) and mobility (e.g. tablet) in the home. The concept also requires a passive demarcation between the home and the provider network and solutions for the OAM of the respective networks in absence of an active box between them. As shown in Figure 2, the power is reduced by eliminating the wireline LAN interfaces on the CPE. This is considered a long term concept, because it assumes new standard interfaces on consumer end-terminals and single-mode fiber infrastructure in the home.

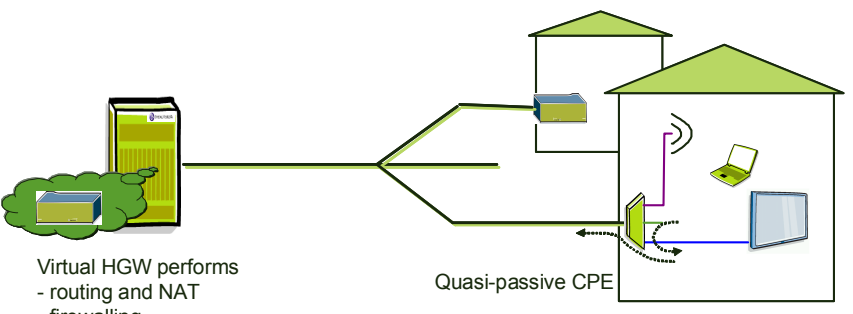

- firewalling

- OAM management

Figure 3. Virtual home gateway and quasi-passive CPE.

Low power electronics and optics innovations are being investigated in various research programmes (e.g. Semiconductor Research Council SRC [9]) and will require that they are adopted by the industry before commercialisation is feasible in the long term. The goal of GreenTouch is not to investigate lower power circuit technologies themselves, but study how they can be used to achieve energy efficiency improvements in the network. Among the many concepts, fault tolerant data transfer and adiabatic switching are realistic in about a decade, because they are feasible with state of the art CMOS manufacturing technologies. Quantum dot automata and other concepts are still further out. Though higher gains have been reported in the literature since they often ignore the interconnections, we assume a factor of three improvement for innovations in electronics and interconnection technology. At the same time more efficient lasers and receivers can be expected for which we assume a factor of two.

\section{SUMMARY AND OUTLOOK}

It is possible to achieve at least $10 \mathrm{x}$ reduction of the power consumption per subscriber in an access network by a combination of different research concepts. On the short term, significant savings can be expected from sleep mode and energy efficient hardware design. On the medium term, node consolidation and virtualization of the home gateway functions can bring additional improvements. On the long term, novel transfer protocols, quasi-passive CPE concepts, and low power electronics and optics will lower the power consumption to 
several hundred milliwatts per subscriber. Considering a doubling of the sustained line rate during busy hour every five year, this results in an efficiency improvement of 100x.

The Wireline Access working group in GreenTouch is currently pursuing more detailed research projects on Green PON, Virtual Home Gateway and quasi-passive CPE, as well as fault tolerance for low power digital electronics in such systems.

\section{ACKNOWLEDGMENT}

The authors would like to thank their colleagues and partners in GreenTouch for valuable discussions and feedback.

\section{REFERENCES}

[1] GreenTouch consortium: www.greentouch.org

[2] D.C. Kilper, G. Atkinson, S.K. Korotky, S. Goyal, P. Vetter, D. Suvakovic, and O. Blume, "Power trends in communication networks," IEEE J. Sel. Quantum. Electronics, vol. 17, no. 2, pp. 275-284, March/April 2011.

[3] ITU-T G.984 Gigabit-capable passive optical network GPON

[4] ITU-T G.987 10-Gigabit-capable passive optical network XG-PON

[5] P. Vetter, and D. Suvakovic, "Research Directions for Low Energy Access Networks (Invited)," ANIC, Toronto, June 2010.

[6] European Commission, Joint Research Centre, "Code of Conduct on Energy Consumption of Broadband Equipment", Version 4, 2011.

[7] IEEE 802.3az Amendment 5: MAC Parameters, PHY layers, and management parameters for Energy Efficient Ethernet, 2010.

[8] L.G. Kazovsky, T. Ayhan, M.R.N. Ribeiro, D. van Veen, "Energy efficient optical wireless residential access/in-house networks," 13th International Conference on Transparent Optical Networks (ICTON), June 2011.

[9] Semiconductor Research Council (SRC): www.src.org 